\title{
In-situ Thermal Testing on Nanostructures in TEM
}

Hua Guo ${ }^{1}$, Will J. Hardy ${ }^{2}$, Panpan Zhou ${ }^{3}$, Douglas Natelson ${ }^{3}$, Jun Lou ${ }^{1}$,

${ }^{1}$. Department of Materials Science and NanoEngineering, Rice University, Houston, Texas, USA

2. Applied Physics Graduate Program, Smalley-Curl Institute, Rice University, Houston, Texas, USA

${ }^{3 .}$ Department of Physics and Astronomy, Rice University, Houston, Texas, USA

As a long-standing problem, sample temperature under electron irradiation in TEM [1] is critical for both scientific interests and technological applications. While, due to the extremely small area of interest, robust method for probing temperature at micron/nano scale is still missing. Efforts were made to measure the temperature rise of the TEM sample, by using carbon nanotube filled with Ga [2], small particles with low melting point [3], MEMS device [4] and EELS spectrum [5]. Here, we demonstrate a new type of microthermometer based on a $\mathrm{VO}_{2}$ nanowire $(\mathrm{NW})$, which derives its fundamental sensing mechanism from the well-known metal-insulator transition in $\mathrm{VO}_{2}$ at $68{ }^{\circ} \mathrm{C}$ [6]. Using this new type of microthermometer, we can not only measure the temperature of small structures but also the heat flow rate generated by a local interaction with the NW, such as the bombardment of high-energy particles.

As shown in Figure 1a, a 59.8-mm-long $\mathrm{VO}_{2} \mathrm{NW}$ with a cross-sectional area of $0.20 \mathrm{~mm}^{2}$ was transferred onto a $\mathrm{Cu}$ TEM grid using a micromanipulator in a focused ion beam (FIB). Following this, a 34.5- mm-long Si NW with a cross-sectional area of $0.31 \mathrm{~mm}^{2}$ was mounted on top of the $\mathrm{VO}_{2} \mathrm{NW}$. At the joints of both the TEM grid and $\mathrm{VO}_{2} \mathrm{NW}$ and the $\mathrm{Si} \mathrm{NW}$ and $\mathrm{VO}_{2} \mathrm{NW}$, electron beam induced $\mathrm{Pt}$ was deposited to join them together, as shown in Figure 1b schematically. Owing to the vacuum of the TEM column, heat convection can be ignored and radiation contributions are also safely negligible. Therefore, our analysis can be limited to one-dimensional thermal conduction. The temperature measurement concept is then based on the fact that as more and more electrons are focused on the extended part of the $\mathrm{VO}_{2} \mathrm{NW}$, the temperature of the local region becomes higher and higher, until the metal-insulator transition (MIT) occurs at $68{ }^{\circ} \mathrm{C}$. The phase transition can be clearly observed in diffraction patterns of the pristine monoclinic I phase (M1) $\mathrm{VO}_{2}$ and the phase transformed rutile phase (R) $\mathrm{VO}_{2}$ at high temperature. The heat flows from the higher temperature part of the NW (under the electron beam) to the lower-temperature part (attached to the TEM grid), resulting in a temperature distribution shown schematically in Figure 1c. When the electron beam is moved onto the Si NW as shown schematically in Figure 1d, a phase transition in $\mathrm{VO}_{2}$ was still expected, but the MIT in $\mathrm{VO}_{2} \mathrm{NW}$ could not be observed directly. On the basis of the previous research [7], it is known that the MIT induces a structural change corresponding to a shrinking of the NW by about $1 \%$. Here, this structual change could be seen as an abrupt lateral jump of the Si and was used as an indication of the MIT. Figure 1d shows the schematic temperature distribution when a high-enough electron beam current was focused onto the $\mathrm{Si} \mathrm{NW}$ to heat the $\mathrm{Si} \mathrm{NW}-\mathrm{VO}_{2} \mathrm{NW}$ joint to $68^{\circ} \mathrm{C}$. As shown in Figure 2, about 1 to 3 $\mu \mathrm{W}$, and $0.62 \mu \mathrm{W}$ of heat is deposited by the incident electron beam into the $\mathrm{VO}_{2} \mathrm{NW}$ and $\mathrm{Si} \mathrm{NW}$, respectively. Owing to its small size the vanadium dioxide nanowire-based microthermometer has a large measurement range and high sensitivity, making it a good candidate to explore the temperature environment of small spaces or to monitor the temperature of tiny, nanoscale objects.

References:

[1] L.W. Hobbs in "Introduction to Analytical Electron Microscopy" (Plenum, 1979). 
[2] Y. Gao, Y. Bando, Nature 415 (2002), p. 599.

[3] T. Brintlinger, Y. Qi, K.H. Baloch, D. Goldhaber-Gordon, J. Cumings, Nano Lett. 8 (2008), p. 582.

[4] L. Shi et al., J. Appl. Phys. 105 (2009), p. 104306.

[5] M. Mecklenburg et al., Science 347 (2015), p. 629.

[6] V. Eyert, Annalen der Physik 11 (2002), p. 650.

[7] Guo, H. et al., Appl. Phys. Lett. 102 (2013), p. 231909.

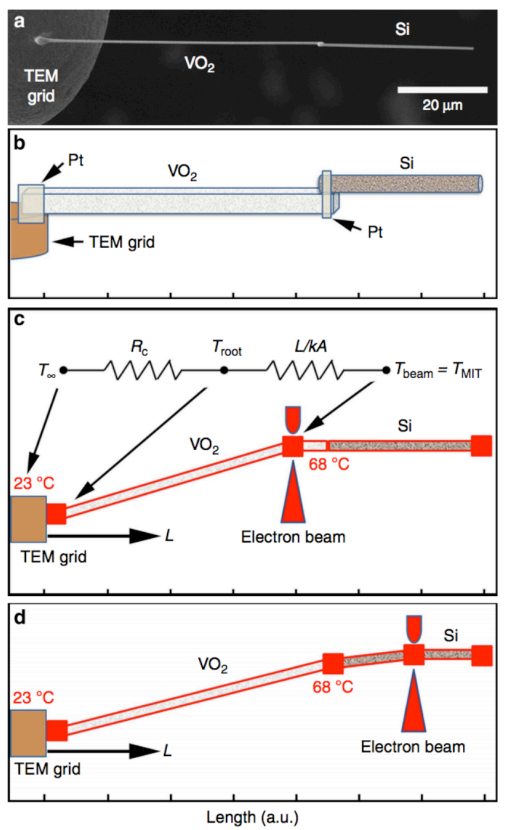

Figure 1. Temperature measurement using a $\mathrm{VO}_{2}$ and Si nanowire in a TEM. (a) Scanning electron microscope picture of the $\mathrm{VO}_{2} \mathrm{NW}$ cantilever, extending from the TEM grid, on top of which a Si NW was mounted. (b) Schematic of the setup in a, showing the location of the electron beam-deposited Pt joints. (c) Diagram of the thermal resistance from the TEM grid to the region under the electron beam and schematic of the temperature distribution within the NWs, when the electron beam was located on $\mathrm{VO}_{2} \mathrm{NW}$. (d) Schematic of the temperature distribution within the NWs when the electron beam was located on Si NW.

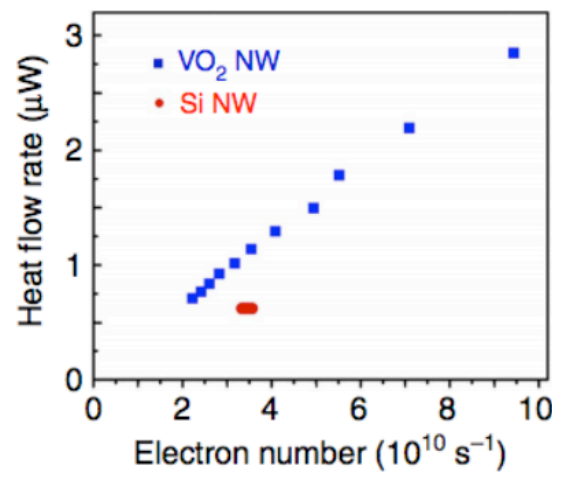

Figure 2. Experimental measurement of heat flow through $\mathrm{Si}$ and $\mathrm{VO}_{2}$ nanowires 